\title{
Universality of young cluster sequences
}

\author{
S. Pfalzner \\ I. Physikalisches Institut, University of Cologne, Zülpicher Str. 77, 50937 Cologne, Germany \\ e-mail: pfalzner@ph1.uni-koeln.de
}

Received 13 March 2009 / Accepted 31 March 2009

ABSTRACT

\begin{abstract}
Aims. Most stars do not form in isolation but as part of a cluster comprising anywhere between a few dozen to several million stars with stellar densities ranging from 0.01 to several $10^{5} M_{\odot} \mathrm{pc}^{-3}$. The majority of these clusters dissolve within 20 Myr. The general assumption is that clusters are born more or less over this entire density range.

Methods. A new analysis of cluster observations is presented.

Results. It demonstrates that, in fact, clustered star formation works under surprisingly tight constraints with respect to cluster size and density.

Conclusions. The observed multitude of cluster densities simply results from snapshots of two sequences evolving in time along pre-defined tracks in the density-radius plane. This implies that the cluster size can actually be used to determine its age.
\end{abstract}

Key words. Galaxy: open clusters and associations: general

\section{Introduction}

The very early phase of star formation is still poorly understood because most stars form in dense clusters embedded within giant molecular clouds (Lada \& Lada 2003; Pudritz 2002) often only observable at infrared wavelengths. If the clusters consist of a thousand stars or more, they also contain massive $\mathrm{O}$ stars that drive the expulsion of gas from the cluster via stellar winds, ionisation and supernovae of early-type stars. This gas expulsion brings the clusters out of dynamical equilibrium, eventually exposing them and leading to a cluster expansion where the majority of stars become unbound (Hills 1980; Goodwin \& Bastian 2006; Baumgardt \& Kroupa 2007; Bastian et al. 2008), eventually turning into field stars. Observations support this picture because young ( $\sim 1 \mathrm{Myr})$ clusters are usually smaller than older ( 20 Myr) clusters, but a systematic and quantitative picture of the cluster expansion itself has so far been lacking. It is this evolution of exposed clusters over the first 20 Myr that we address here. It is a time-span of special interest because it is precisely the period when planetary systems are developing.

The observed densities of young star clusters range from less than 0.01 to several $10^{5} M_{\odot} \mathrm{pc}^{-3}$ as Fig. 1 shows (a list of cluster properties is given in Table A.1: Figer 2008; Wolff et al. 2007; Borissova et al. 2008), where the determination methods of the masses and radii from the literature sources are also explained). Here only clusters more massive than $1000 M_{\odot}$ (massive clusters) are included, since the size and density of clusters containing only a few dozen stars are poorly determined. The wide variety of cluster densities has lead to the general assumption that clusters are also formed over this entire density range. As a result of the gas expulsion, a rapid dissolution of the cluster because of their super-virial velocity dispersion (Hills 1980; Kroupa 2005; Goodwin \& Bastian 2006; Gieles \& Bastian 2008) is then expected.

\section{Results}

When looking closely at the same clusters as in Fig. 1 in a different way by plotting instead the density as a function of the

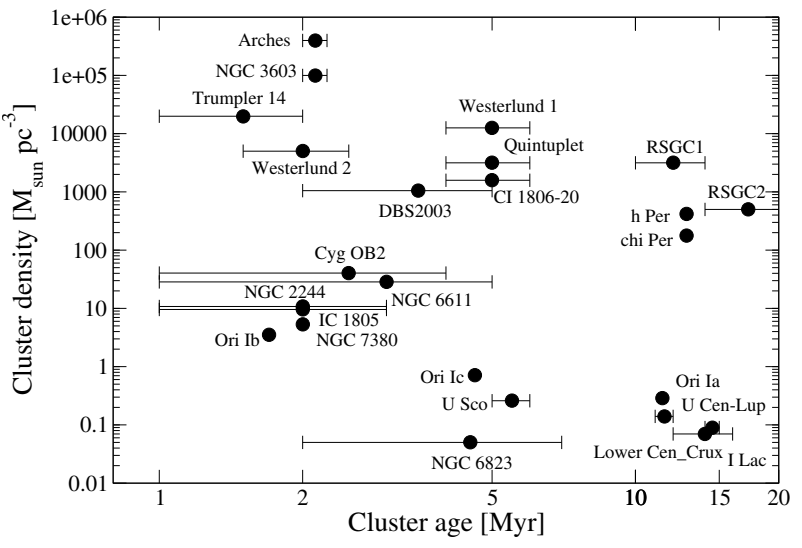

Fig. 1. Cluster density as a function of age for clusters more massive than $10^{3} M_{\odot}$. The values were taken from Figer (2008), Wolff (2007), and Borissova et al. (2008) and references therein. See Table A.1 as well for a discussion of the error bars.

cluster radius $R$ (definitions for the individual data points are given in the Appendix) leads to the remarkably simple structure in Fig. 2. Apart from clusters existing in two groups, what this plot unambiguously shows is that these evolve along two welldefined tracks in the density-radius plane, strongly suggesting a bi-modal cluster evolution. Moreover, as can be seen in Fig. 2, the two classes of clusters - in the following called starburst and leaky clusters (the terminology will be explained later) each start from unique points in the mass-radius plane and develop at approximately the same speed along these two tracks. All clusters shown originate from one of these birth points in mass and radius.

This is not so surprising for starburst clusters since their relatively similar mass has already led to speculations that they might have a common origin. For leaky clusters, however, the prevailing view is that young clusters form more or less at arbitrary density and somehow dissolve afterwards on timescales of 20 Myr. However, the new representation of already known 


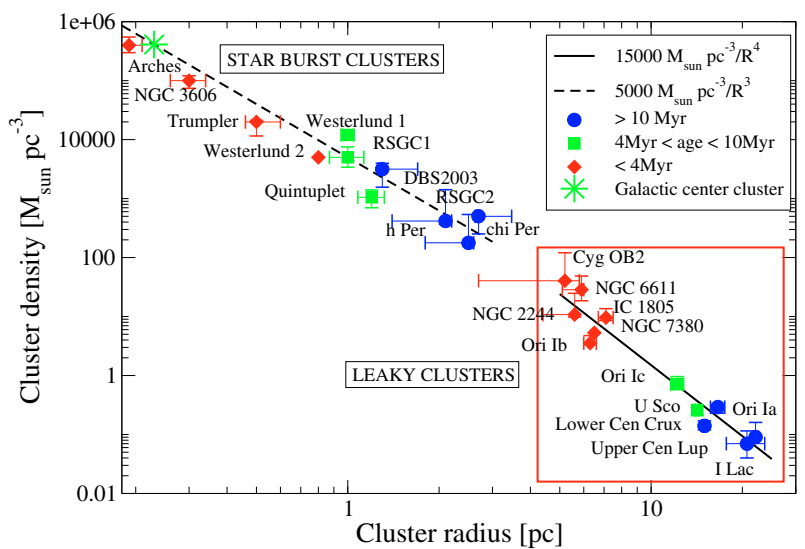

Fig. 2. Cluster density as a function of cluster size for clusters more massive than $10^{3} M_{\odot}$. The values were taken from Figer (2008), Wolff et al. (2007) and Borissova et al. (2008) and references therein. See although Table A.1.

cluster parameters in Fig. 2 shows the common history of these clusters for the first time. The single track and sequential nature of the data points means that, contrary to the usual viewpoint, these clusters also start out with the same mass density and radial extent (and therefore mass) and all expand in the same way.

Here a word about determinating of radii and densities in the different sources is nessesary. All starburst cluster data, apart from that for DBS 2003, were taken from Figer (2008). The data for DBS 2003 were taken from Borissova et al. (2008). In Figer (2008) the radius gives the average projected separation from the centroid position. The total cluster mass is given by extrapolating down to a lower mass cut-off of 1 solar mass, assuming a Salpeter initial mass function and an upper mass cut-off of 120 solar masses (exceptions from this method are given in Figer 2008). The total mass of the cluster was determined by assuming that the stars in the mass range $6-12 M_{\odot}$ constitute $5.5 \%$ of the total mass of the complete population of stars spanning the range $0.1-100 M_{\odot}$. They compared their values with other massdetermining methods by Slesnick et al. (2002) and Weidner \& Kroupa (2006) and found good agreement. In Borissova et al. the radius was defined by distance where the density profile exceeds twice the standard deviation of the surface density in the surrounding field. All values for the leaky clusters were taken from Wolff et al. (2007). There the radius was determined as the median distance in degrees of the B stars in the sample from the centre of the cluster, so that, although not all cluster sizes were determined exactly in the same way, for each subset - the starburst clusters and the leaky clusters - the cluster radii were determined fairly consistently. The density was in all cases defined as $\rho=3 M_{\mathrm{c}} / 4 \pi R^{3}$, so it is extremely unlikely that the result is an artefact of the applied method.

Quantitative analysis of Fig. 2 shows that, as the leaky clusters expand, they do not simply diffuse (which would give an $R^{-3}$-dependence), but the cluster density decreases as $R^{-4}$. This means that the cluster loses mass via various processes such as outgassing, stellar mass loss, tidal evaporation, and escapers created through three-body encounters close to its centre. The relative importance of these mass-loss processes will need clarification in the future. As a direct consequence of the common history of these clusters, one can now determine the cluster size as a function of cluster age and finds a $t_{\mathrm{c}}^{0.6-0.7}$-dependence. As demonstrated in Fig. 3, the expansion velocity is $\sim 2 \mathrm{pc} / \mathrm{Myr}$ in the early phases and drops to $\sim 1 \mathrm{pc} / \mathrm{Myr}$ at cluster ages $\sim 20 \mathrm{Myr}$. In Fig. 3 it is shown that comparing simulations of clusters

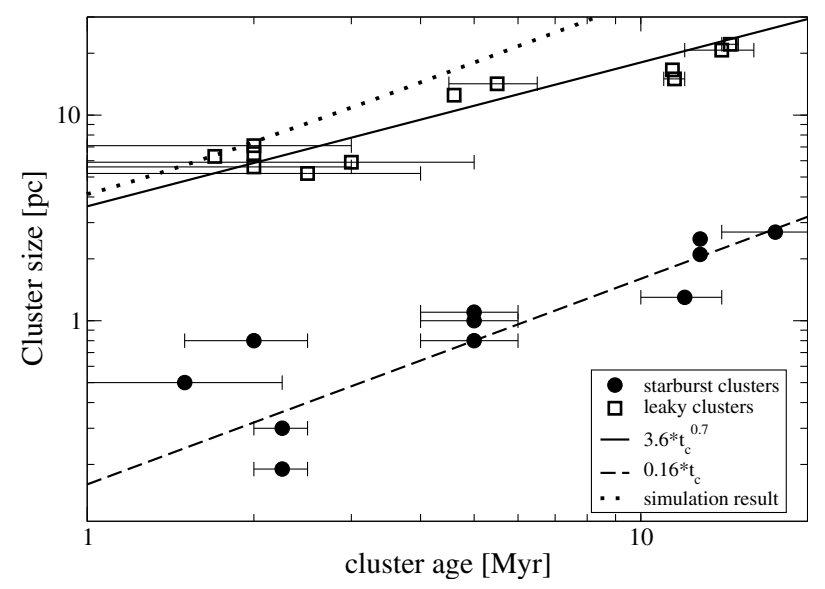

Fig. 3. Cluster size as a function of cluster age for the same clusters in Fig. 2. The squares correspond to the leaky clusters and the circles to the starburst clusters. The dotted line indicates simulation results by Olczak (2009) for an ONC-like cluster with 75\% gas expulsion. The drawn and the dashed lines are not fits but just there to guide the eye.

expanding due to gas expulsion showing similar expansion velocity (Olczak 2008) indicates that $\sim 75 \%$ of the gas is expelled, though more detailed studies are required to confirm this. However, this would already suggest that all leaky clusters shown in Fig. 2 formed with the approximately the same star formation efficiency of $\sim 25 \%$.

It is thought that clusters and associations are formed with mass functions that are powerlaws with index -2 . However, the results described here imply that the leaky clusters form in a narrow mass range $\left(3.6<\log M_{\mathrm{c}}<4.4\right)$ and develop quickly within $20 \mathrm{Myr}$ to lower masses $\left(3.3<\log M_{\mathrm{c}}<3.6\right)$. The small sample size currently does not allow further constriction of the primordial mass function for loss clusters.

The situation is simpler for the starburst clusters. They all appear to be born with a cluster density of at least $10^{5} M_{\odot} \mathrm{pc}^{-3}$ (red data points in the top left corner of Fig. 2) and then simply diffuse without further mass loss, i.e. a $R^{-3}$ dependence. In fact the expansion proceeds more or less linearly in time, i.e. as $R \sim t_{\mathrm{c}}$ (see Fig. 3). Having established a common history, one can easily deduce an expansion velocity of $0.1-0.2$ pc/Myr. From this expansion velocity, it should be possible to determine the star formation efficiency through numerical simulations in the future. A possible reason for the expansion velocity in star-burst clusters to be lower than in leaky clusters is that the potential well is too deep for the ionised gas to leave the cluster quickly (Kroupa 2008). This means a much higher star formation efficiency for starburst clusters than for the massive clusters.

Figure 2 shows an additional feature, namely the location of the Galactic centre cluster. Since the Galactic centre harbours a massive black hole, one would expect its dynamics to differ considerably from starburst clusters. However, it lies directly on the starburst cluster track close to the data point of Arches. Given that the Galactic centre cluster is older than the Arches cluster, it follows that it must have started out at a higher initial mass and/or expanded more slowly.

If the above systematics is true, then no clusters should exist with $R>2$ pc ages ages $<4$ Myr that possess masses higher than several $10^{4} M_{\odot}$. An interesting consequence of the sequential nature of cluster development is that the radius of the cluster can actually be used to determine its age. Cluster age determination is notoriously problematic, especially for very young clusters, where the uncertainty of the tracks of pre-main sequence star development can lead to very different age estimates. The 


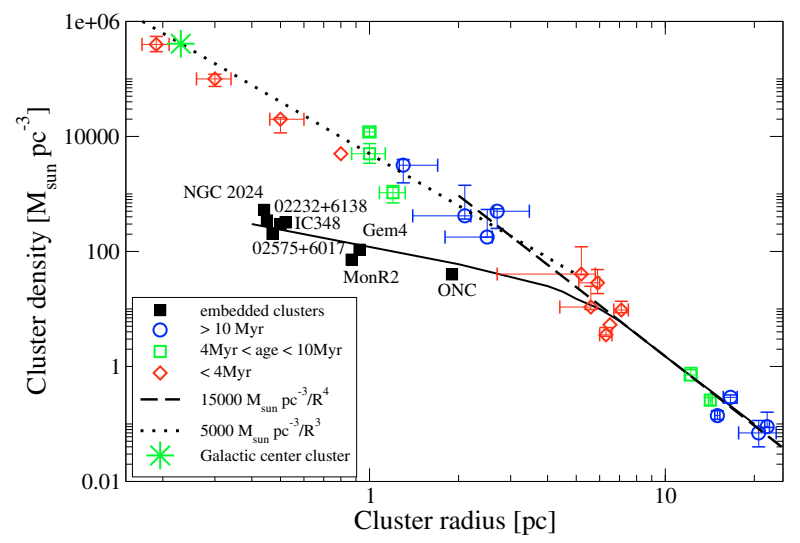

Fig. 4. Same as Fig. 2 but here the cluster density of embedded clusters with more than observed 200 members are also shown. The values were taken from Lada \& Lada (2003).

density-radius relation defined here might be an easier and potentially more accurate method for cluster age determination. Clusters with a radius between 2-4 pc pose a special problem. Only the distinction between very young leaky clusters (<4 Myr) and old starburst clusters ( $>10 \mathrm{Myr}$ ) will require additional information due to their similar radii. However, as one can expect that in older starburst clusters some red supergiants are present, telling them apart is probably not too difficult.

How do embedded clusters fit into this picture? Fig. 4 again shows the cluster density as a function of size but also includes embedded clusters. The embedded clusters were selected from the data by Lada \& Lada (2003) with the restriction that at least 200 cluster stars were detected. The embedded clusters are not located on the main evolutionary sequence but form their own side-arm off the leaky cluster sequence below its extension to younger ages. It joins up when the embedded clusters become exposed after having reached a size of $\sim 2-4 \mathrm{pc}$ at an age of $>1$ Myr. A further argument for the sequential nature of the cluster development is that the ONC is likely to be expanding (Kroupa et al. 2001), hence evolving along a time-density track. In other words, a relatively sparse cluster like Upper Cen Lup must once have been in a state similar to the Orion Nebular Cluster now the densest nearby cluster (see Fig. 4). Note as well that Ori Ib, Ori Ic, and Ori Ia form a temporal sequence of once leaky clusters with initial densities as high as the ONC. The initial stellar mass content of leaky clusters at the end of the embedded phase is at least $3500 M_{\odot}$. with a radius exceeding $\sim 4 \mathrm{pc}$,

One would expect a similar side-arm for the starburst clusters, but so far no such embedded starburst clusters have been observed. However, this is not surprising since they would be very small $(R<0.1 \mathrm{pc})$ and located either close to the Galactic centre (at a distance of $\sim 8 \mathrm{kpc}$ ) or inside spiral arms within the Galactic plane. In addition, starburst clusters are intrinsically rare, and the embedded phase very short, so not many are expected to be found.

\section{Discussion and conclusions}

The above results imply that star formation occurs only under an extremely limited set of conditions, and may therefore require a fundamental revision of its theories in our Galaxy, possibly including our own solar system. The "leaky clusters" could also be known as "mass-loss clusters", as this is what distinguishes them from starburst clusters.". For this leaky cluster density regime, recent simulations (Küpper et al. 2008) have found that the dynamics of embedded clusters show a strong correlation between
Table A.1. Properties of clusters and associations more massive than $10^{3} M_{\odot}$ in the Galaxy.

\begin{tabular}{|c|c|c|c|c|c|}
\hline Identification & $\begin{array}{c}\text { Distance } \\
{[\mathrm{pc}]}\end{array}$ & $\begin{array}{c}\text { Age } \\
{[\mathrm{Myr}]}\end{array}$ & $\begin{array}{c}\log \left(M_{\mathrm{c}}\right) \\
{\left[M_{\odot}\right]}\end{array}$ & $\begin{array}{l}\text { size } \\
{[\mathrm{pc}]}\end{array}$ & $\begin{array}{c}\log \left(\rho_{\mathrm{c}}\right) \\
{\left[M_{\odot} \mathrm{pc}^{-3}\right]}\end{array}$ \\
\hline $\operatorname{Arches}^{1}$ & $8_{-1}^{+1}$ & $2-2.5$ & 4.3 & $0.19_{-0.03}^{+0.03}$ & $5.6_{-1}^{+1}$ \\
\hline NGC $3603^{1}$ & $7.6_{-1}^{+1}$ & $2-2.5$ & 4.1 & $0.3_{-0.04}^{+0.04}$ & $5.0_{-0.1}^{+0.1}$ \\
\hline Trumpler $14^{1}$ & $2.8_{-0.2}^{+0.6}$ & $<2$ & 4.0 & $0.5_{-0.04}^{+0.1}$ & $4.3_{-0.3}^{+0.05}$ \\
\hline Westerlund $1^{1}$ & $3.55_{-0.01}^{+0.17}$ & $4-6$ & 4.7 & $1.0^{+0.05}$ & $4.1_{-0.03}^{+0.06}$ \\
\hline Westerlund $2^{1}$ & 2.8 & $1.5-2.5$ & 4.0 & 0.8 & 3.7 \\
\hline RSGC $1^{1}$ & $5.83_{-0.78}^{+1.9}$ & $10-14$ & 4.5 & $1.3_{-0.04}^{+0.04}$ & $3.5_{-0.3}^{+0.1}$ \\
\hline Quintuplet $^{1}$ & $8_{-1}^{+1}$ & $4-6$ & 4.3 & $1.0^{+0.13}$ & $3.7_{-0.2}^{+0.7}$ \\
\hline DBS2003² & $7.9_{-1}^{+1.2}$ & $2-5$ & 3.8 & $1.2_{-0.5}^{+0.18}$ & $3.05_{-0.3}^{+0.5}$ \\
\hline RSGC $2^{1}$ & $5.83_{-0.78}^{+1.91}$ & $14-21$ & 4.6 & $2.7_{-0.77}^{+0.77}$ & $2.7_{-0.03}^{+0.05}$ \\
\hline$\chi \mathrm{Per}^{3}$ & $2.34_{-0.5}^{+0.1}$ & 12.8 & 4.1 & $2.5_{-0.7}^{+0.1}$ & $2.61_{-0.05}^{+0.5}$ \\
\hline h $\mathrm{Per}^{3}$ & $2.34_{-0.5}^{+0.1}$ & 12.8 & 4.2 & $2.1_{-0.7}^{+0.1}$ & $2.25_{-0.05}^{+0.5}$ \\
\hline $\mathrm{CYg} \mathrm{OB} 2^{3}$ & $1.74_{-0.5}^{+0.2}$ & $1-4$ & 4.4 & $5.2_{-2.5}^{+0.06}$ & $1.61_{0.4}^{+0.02}$ \\
\hline NGC $6611^{3}$ & $1.995_{-0.25}^{+0.01}$ & $1-5$ & 4.4 & $5.9_{-0.8}^{+0.1}$ & $1.45_{0.11}^{+0.22}$ \\
\hline NGC $2244^{3}$ & $1.88_{-0.4}$ & $1-3$ & 3.9 & $5.6_{-1.2}$ & $1.03^{+0.33}$ \\
\hline IC $1805^{3}$ & $2.34_{-0.1}^{+0.1}$ & $1-3$ & 4.2 & $7.1_{-0.3}^{+0.3}$ & $0.98^{+0.03}$ \\
\hline Ori $\mathrm{Ib}^{3}$ & $0.363_{-0.2}^{+0.2}$ & 1.7 & 3.6 & $6.3_{-0.3}^{+0.3}$ & $0.55_{-0.02}^{+0.11}$ \\
\hline NGC $7380^{3}$ & 3.73 & 2 & 3.8 & 6.5 & 0.72 \\
\hline Ori $\mathrm{Ic}^{3}$ & $0.398_{-0.2}^{+0.2}$ & 4.6 & 3.8 & $12.5_{-0.6}^{+0.6}$ & $-0.15_{-0.01}^{+0.12}$ \\
\hline Ori Ia ${ }^{3}$ & $0.380_{-0.2}^{+0.2}$ & 11.4 & 3.7 & $16.6_{-0.9}^{+0.9}$ & $-0.54{ }_{-0.09}^{+0.05}$ \\
\hline $\mathrm{U} \mathrm{Sco}^{3}$ & $0.144_{-0.003}^{+0.003}$ & $5-6$ & 3.5 & $14.2_{-0.2}^{+0.2}$ & $-0.59_{-0.01}^{+0.06}$ \\
\hline Lower Cen-Crux ${ }^{3}$ & $0.116_{-0.002}^{+0.002}$ & $11-12$ & 3.3 & $15.0_{-0.3}^{+0.3}$ & $-0.85_{-0.01}^{+0.05}$ \\
\hline Upper Cen-Lup $2^{3}$ & $0.142_{-0.002}^{+0.002}$ & $14-15$ & 3.6 & $22.1_{-0.4}^{+0.4}$ & $-1.05_{-0.01}^{+0.01}$ \\
\hline I Lac $2^{3}$ & $0.368^{+0.06}$ & $12-16$ & 3.4 & $20.7_{-3}^{+3}$ & $-1.15_{-0.17}^{+0.2}$ \\
\hline
\end{tabular}

${ }^{1}$ Figer (2008), and references therein; ${ }^{2}$ Borissova et al. (2008); ${ }^{3}$ Wolff et al. (2007), and references therein.

the kinetic energy of the cluster stars and that of stars leaving the cluster, leading to a common sequence. This prediction might well be linked to the results described herein.

The embedded cluster side-arm implies that many of the older clusters (like Ori Ia-c, Upper Sco, I Lac, etc.) must have gone through a phase like the ONC is in today. Thus the ONC can serve as model cluster for both the earlier phase of older leaky clusters and the future of the younger embedded clusters. Results for the ONC therefore serve as a template for a multitude of other clusters. For example, observations and theory indicate that the central density in the ONC is so high that encounters between young disc-surrounded stars might affect the protoplanetary discs (Herbig \& Terndrup 1986; Pfalzner et al. 2006) and changes in the disc properties are likely to have direct consequences on the ongoing planet formation process.

Finally the proposed reordering immediately raises the question of the origin of these two distinct cluster groupings. The critical parameter is possibly the higher density, but could equally be the density-temperature ratio or different types of turbulence, etc. The obvious task for the near future is to understand why these specific conditions are favoured in our Galaxy and whether the same applies in other spiral galaxies, too.

\section{Appendix A}

In Table A. 1 the errors in age result from (a) their deduction from theoretical pre-main-sequence star tracks for young stars, which 
Table A.2. Properties of embedded clusters containing more than 200 observed stars.

\begin{tabular}{rcccc}
\hline \hline Identification & $\begin{array}{c}\text { distances } \\
{[\mathrm{pc}]}\end{array}$ & $\begin{array}{c}M_{\mathrm{c}} \\
{\left[M_{\odot}\right]}\end{array}$ & $\begin{array}{c}\text { Size } \\
{[\mathrm{pc}]}\end{array}$ & $\begin{array}{c}\log \left(\rho_{\mathrm{c}}\right) \\
{\left[M_{\odot} \mathrm{pc}^{-3}\right]}\end{array}$ \\
\hline $02232+6138^{1}$ & 2.4 & 130 & 0.91 & 2.23 \\
$02575+6017^{1}$ & 2.4 & 150 & 1 & 2.17 \\
$\mathrm{IC} 348^{1}$ & 0.32 & 160 & 1 & 2.20 \\
ONC $^{1}$ & 0.45 & 1100 & 3.8 & 1.30 \\
$\mathrm{NGC} 2024^{1}$ & 0.4 & 180 & 0.88 & 2.42 \\
MonR2 $^{1}$ & 0.8 & 340 & 1.85 & 1.72 \\
\hline
\end{tabular}

${ }^{1}$ Lada \& Lada (2003), and references therein.

show large discrepancies; (b) the age of the cluster is found by averaging over the so deduced ages of the single stars; and (c) the age determination depends on the knowledge of the distance to the cluster - a parameter that is often poorly constrained. In cases where there is no error bar this does not mean that the age is accurately known, but rather that the authors did not give any error value. Usually there are no error values quoted for the mass or the density of the cluster, nor is the cluster size consistently defined for the different clusters. An error of a factor of 2 is probably realistic and can be expected to be even larger for the more distant clusters, so for this and the following figures one should always keep in mind that the errors of these observed values are rather large.

\section{References}

Bastian, N., Gieles, M., Goodwin, S. P., et al. 2008, MNRAS, 389, 223 Baumgardt, H., \& Kroupa, P. 2007, MNRAS, 380, 1589

Borissova, J., Ivanov, V. D., Hanson, M. M., et al. 2008, A\&A, 488, 151

de Grijs, R., Lee, J. T., Mora Herrera, M. C., et al. 2003, New Astronomy, 8, 155 Figer, D. F. 2008, in Massive Stars as Cosmic Engines, Proc. IAU Symp., 250, 247

Gieles, M., \& Bastian, N. 2008, A\&A, 482, 165

Goodwin, S. P., \& Bastian, N. 2006, MNRAS, 373, 752

Herbig, G. H., \& Terndrup, D. M. 1986, ApJ, 307, 609

Hills, J. G. 1980, ApJ, 235, 987

Kroupa, P. 2005, in The Three-Dimensional Universe with Gaia, Proc. of Gaia Symp., ed. C. Turon, K. S. O'Flaherty, \& M. A. C. Perryman, ESA SP-576, 629

Kroupa, P. 2008, in Proc. of Mass Loss from Stars and the Evoltution of Stellar Clusters, ed. A. de Koter, L. Smith, \& R. Waters, San Francisco, ASP Conf. Ser., 388, 271

Kroupa, P., Aarseth, S., \& Hurley, J. 2001, MNRAS, 321, 699

Küpper, A. H., Kroupa, P., \& Baumgardt, H. 2008, MNRAS, 389, 889

Lada, C. J., \& Lada, E. A. 2003, ARA\&A, 41, 57

Olczak, C. 2008, private communication

Pfalzner, S., Olczak, C., \& Eckart, A. 2006, A\&A, 454, 811

Pudritz, R. E. 2002, Science, 295, 68

Slesnick, C. L., Hillenbrand, L. A., \& Massey, P. 2002, ApJ, 576, 880

Weidner, C., \& Kroupa, P. 2006, MNRAS, 365, 1333

Wolff, S. C., Strom, S. E., Dror, D., Lanz, L., \& Venn, K. 2007, Astron. J., 132, 749 